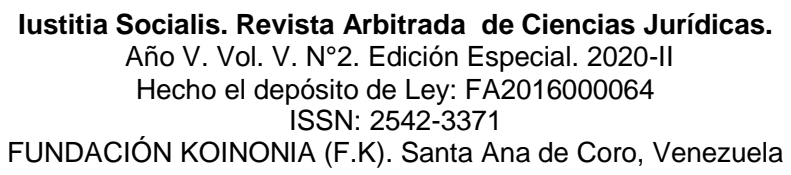

FUNDACIÓN KOINONIA (F.K). Santa Ana de Coro, Venezuela

Alison Joyce Zapata Benavides; Julio César de Jesús Arrias-Añez; Juan Carlos Aradia Zambrano

http://dx.doi.org/10.35381/racji.v5i2.1057

\title{
El delito del peculado y su impacto socioeconómico en el Ecuador
}

The crime of embezzlement and its socioeconomic impact in Ecuador

\author{
Alison Joyce Zapata-Benavides \\ zapataalison55@gmail.com \\ Universidad Regional Autónoma de Los Andes, Quevedo \\ Ecuador \\ https://orcid.org/0000-0002-4026-1470 \\ Julio César de Jesús Arrias-Añez \\ uq.julioarrias@uniandes.edu.ec \\ Universidad Regional Autónoma de Los Andes, Quevedo \\ Ecuador \\ https://orcid.org/0000-0001-5259-9359 \\ Juan Carlos Aradia-Zambrano \\ uqjuanarandia@uniandes.edu.ec \\ Universidad Regional Autónoma de Los Andes, Quevedo \\ https://orcid.org/0000-0003-2624-0427
}

Recibido: 15 de septiembre de 2020

Revisado: 20 de octubre de 2020

Aprobado: 04 de diciembre de 2020

Publicado: 11 de diciembre de 2020 
lustitia Socialis. Revista Arbitrada de Ciencias Jurídicas.

Año V. Vol. V. N². Edición Especial. 2020-II

Hecho el depósito de Ley: FA2016000064

ISSN: 2542-3371

FUNDACIÓN KOINONIA (F.K). Santa Ana de Coro, Venezuela

Alison Joyce Zapata Benavides; Julio César de Jesús Arrias-Añez; Juan Carlos Aradia Zambrano

\title{
RESUMEN
}

El objetivo de la investigación fue desarrollar un análisis crítico jurídico sobre el delito de peculado y su repercusión económica y social para fundamentar propuestas relacionadas con el tratamiento de este delito en el sistema jurídico del Ecuador. Se procesó en razón del método analítico - sintético. El delito de peculado ocasiona una magnitud de daño gravosa, por su repercusión socioeconómica, al generar una pérdida de valores éticos económicos por parte de los infractores y por las consecuencias que afectan a la colectividad que ven como su erario se deprecia y deprime y con esto el funcionamiento eficaz de estamentos imprescindibles para el desarrollo del buen vivir van en detrimento, lo cual redunda en la insatisfacción de necesidades básicas para la población, coyuntura que podría llevarnos a un Estado fallido y a la transgresión del Estado de Derecho.

Descriptores: Corrupción; crimen; derecho penal. (Palabras tomadas del Tesauro UNESCO).

\begin{abstract}
The objective of the research was to develop a critical legal analysis on the crime of embezzlement and its economic and social repercussions to support proposals related to the treatment of this crime in the Ecuadorian legal system. It was processed by reason of the analytical-synthetic method. The crime of embezzlement causes a serious magnitude of damage, due to its socioeconomic repercussions, by generating a loss of ethicaleconomic values on the part of the offenders and due to the consequences that affect the community who see their treasury depreciate and depress and with As a result, the effective functioning of essential elements for the development of good living are detrimental, which results in the dissatisfaction of basic needs for the population, a situation that could lead to a failed State and the violation of the rule of law.
\end{abstract}

Descriptors: Corruption; crime; criminal law. (Words taken from the UNESCO Thesaurus). 


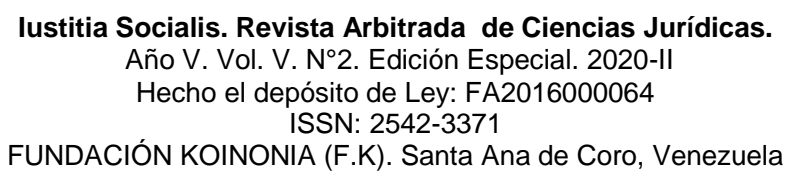

Alison Joyce Zapata Benavides; Julio César de Jesús Arrias-Añez; Juan Carlos Aradia Zambrano

\section{INTRODUCCIÓN}

La corrupción institucionalizada en el Ecuador desde las esferas del poder es quizá el problema más concurrente que tiene éste país desde el ámbito político. A diferencia de estar en los primeros lugares en los indicadores de ciencia, tecnología e innovación, siempre encabeza los primeros lugares en corrupción, pero no cualquier corrupción, sino la de "cuello blanco", como la definía Edwin H. Sutherland, sociólogo y criminólogo estadounidense (Jaramillo, 2019).

Gracias a la conectividad y a la viralidad que tienen las noticias en la internet, se están descubriendo tramas de corrupción en donde están involucrados representantes de los entes del Estado en todos los niveles como lo son el central, regional y local que ejercen la gestión de entres tan importantes para el desarrollo del buen vivir como es el caso del Instituto Ecuatoriano de Seguridad Social (IESS) al cual están adscritos los principales centros de salud públicos que atienden las necesidades de la población en la materia.

En este sentido, para ser considerado un acto administrativo como corrupción en afectación del patrimonio nacional, (Encalada-Hidalgo, 2014), como que, en los Estados constitucionales, no solo se debe trabajar desde la base del derecho penal, sino que como "requisito sine qua non para el establecimiento de una sanción penal; ahora se exige además que se cumpla con el principio de lesividad" (p. 103), lo cual conduce a deducir, no solo la necesidad de contar con indicios o suposiciones de corrupción, sino, que estas deben ser justamente demostradas y comprobadas ante el órgano judicial correspondiente, para ser calificada como delito, por tal razón, es necesario que la administración de justicia del Ecuador, afine los detalles para entablar orden y ley, ante los delincuentes de cuello blanco, por cuanto pueden valerse de procedimiento administrativos que amparen su delito, es decir, para generar una percepción de legalidad, así como vacíos jurídicos en la ley.

En este escenario, se han reseñado varias noticias de carácter alarmante relacionadas con la detección de irregularidades en los procedimientos de adjudicaciones directas llevados a cabo durante la emergencia sanitaria de acuerdo a los lineamientos 


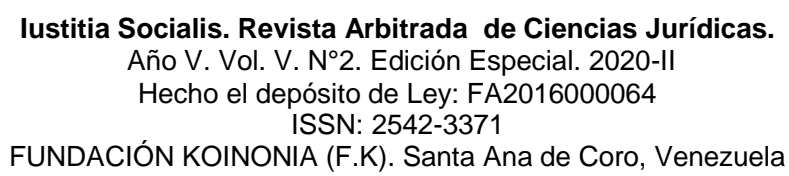

Alison Joyce Zapata Benavides; Julio César de Jesús Arrias-Añez; Juan Carlos Aradia Zambrano

direccionados por el Comité de Operaciones de Emergencia Nacional (COE) en virtud del Estado de Excepción dictado por el presidente de la república para la adquisición de insumos y equipos médicos, así como la construcción de centros de salud para combatirla pandemia generada por el virus del COVID-19.

Al respecto, de acuerdo a una nota periodística del (El Universo, 2020), "varias instituciones del Estado como El Instituto Ecuatoriano de Seguridad Social (IESS) a través de los procedimientos desarrollados por adjudicación directa por medio de sus respectivas autoridades, incurrió en el delito de peculado debido a los sobreprecios de hasta el $9000 \%$ en la adquisición de medicamentos., coyuntura que representa una acción típica, antijurídica y culpable que la Ley de Contratación pública prohíbe expresamente, resaltando la compra de insumos médicos, en un contrato de más de $\$$ 10 millones en favor de la empresa Almantop S. A."

Un claro ejemplo se da en la provincia de Manabí, cuando en el año 2016 tuvo que soportar un terremoto de magnitudes apocalípticas, quedando destruida su infraestructura vial y hospitalaria, al efecto se destinaron 124 millones de dólares para su reconstrucción, sin embargo, muy poco se ha avanzado y el dinero ya se ha gastado y la población manabita de Pedernales sufre el déficit hospitalario y por ende la falta de atención ante las emergencias de salud.

El cantón Pedernales que tiene una población de 88.171 habitantes, el Servicio de Contratación de Obras, hizo público en el Portal De Compra Públicas, el proceso de licitación LICO-SECOB-004-2019 para la construcción del Hospital Básico de Pedernales (Jalil-Ponce, 2019), en el cual se invocó la participación de personas naturales y jurídicas, nacionales o extranjeras, a través de un proceso transparente donde se garantizaría el cumplimiento de todas las pautas establecidas en todos los ordenamientos jurídicos y administrativos relativos a la materia donde no se permitiría la corrupción, que han sido denunciados en la Fiscalía General del Estado.

La colectividad es la principal afectada por el retraso en el avance de tan esperada obra, si se toma en cuenta que han transcurrido cuatro largos años desde que ocurrió el 
terremoto, y la población de la localidad necesita con urgencia atención en el área de salud y la obra ha entrado en la más grande polémica, porque ha sido intervenida por la contraloría que ha descubierto indicios penales de responsabilidad, en donde se involucran sendos personajes del gobierno.

Así las cosas, innumerables son los casos por corrupción, sin embargo, pocos son los resultados, pero ¿qué es lo que detiene el camino de la justicia?, las normas penales están, las instituciones creadas para sancionar y ejecutar penas también lo están, sin embargo, es una invención de nunca acabar el reparto, abuso y robo descarado de los fondos estatales.

Por esto, la actualidad y trascendencia del presente trabajo es vital, ya que los asambleístas, ministros, alcaldes, prefectos y un sin número de autoridades administrativas y de elección popular han sido salpicados por escándalos de corrupción en torno a compra de medicinas y mala administración de fondos públicos a raíz de la crisis de salud que está pasando en torno al COVID-2019. Aunque el problema era latente, con la actual crisis se ha puesto de relieve el costo económico que tienen este delito para el Estado, pues es dinero público que va a manos privadas, por medio de la corrupción, dinero que debería ser invertirlo en educación, infraestructura, salud, entre otros, lamentablemente no sucede aquello, las instituciones están secuestradas por la corrupción. En atención a la problemática previamente expuesta se formula el siguiente objetivo general:

Desarrollar un análisis crítico jurídico sobre el delito de peculado y su repercusión económica y social para fundamentar propuestas relacionadas con el tratamiento de este delito en el sistema jurídico del Ecuador. 


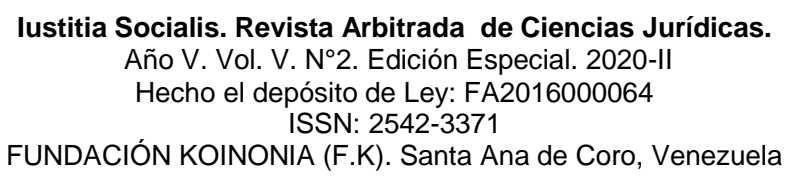

Alison Joyce Zapata Benavides; Julio César de Jesús Arrias-Añez; Juan Carlos Aradia Zambrano

\section{MÉTODO}

El trabajo investigativo se procesó en razón del método analítico - sintético, analizándose el objeto investigativo (delito de peculado), desarrollándose el análisis de los contextos argumentativos para conformarse una proposición sintética como reflexión del proceso investigado (Lopera-Echavarría, et al., 2010).

El estudio es una investigación descriptiva documental con diseño bibliográfico, recurriéndose a una población conformada por leyes, documentos, trabajos de investigación académica, investigaciones periodísticas, como parte de la argumentación analizada, develándose a través de la técnica de análisis de contenido, los aspectos centrales que conformaron el articulado teorético - jurídico como resultado propositivo del derecho ecuatoriano.

\section{RESULTADOS}

El Código Orgánico Integral Penal de 2014 en el art. 278 tipifica el peculado como conducta punible e impone una pena privativa de libertad de diez a trece años (Asamblea Nacional de la República del Ecuador, 2014). Es menester puntualizar que "el delito de peculado se refiere a la malversación del dinero del Estado, es decir, de los fondos públicos. Es el enriquecimiento indebido de un funcionario público o alguien que está encargado del servicio público" (Quintero, 2018, p. 1), el cual aprovecha su condición para apropiarse del capital, y este es un daño gravísimo a la sociedad y a la economía del país porque por cuanto forma parte del erario nacional y está orientado a la satisfacción de necesidades de la población en cuanto a educación, salud, alimentación, seguridad social, trabajo entre otras, esa es la mayor afectación a los ciudadanos.

El peculado es quizá el delito de comisión con mayor aplicación en el contexto de la administración pública, por cuanto este es esencialmente un "problema político" (KunickaMichalska, 2017), ante dicha coyuntura la investigación propone explorar acerca de este tipo penal y su tratamiento en el ordenamiento jurídico ecuatoriano. Como investigadora es obligación hacer un aporte crítico a la investigación, y la mejor vía es a través de este 


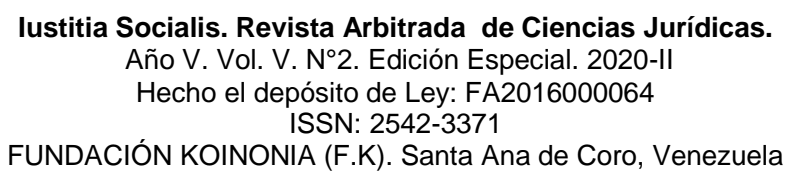

Alison Joyce Zapata Benavides; Julio César de Jesús Arrias-Añez; Juan Carlos Aradia Zambrano

artículo científico sobre de este estado de inercia, que tiene atado de pies y manos a la nación ecuatoriana, cuestión que limita la prosperidad por efectos de tanta corrupción generalizada y que se deja impune y permisible por parte del Estado central.

En este contexto, es oportuno resaltar que la perpetración de este tipo penal se ejecuta de manera recurrente en el marco de procedimientos licitatorios y de adjudicaciones directas, surgiendo una serie de irregularidades llevadas a cabo con ocasión a los procesos en los cuáles se necesitan la adquisición de equipos e insumos médicos necesarios con urgencia para tratar emergencias sanitarias como lo representa la pandemia generada por el COVID 19.

Conforme a la Ley Orgánica del Sistema Nacional de Contratación Pública de Ecuador de 2009, en su artículo 57, se devela el procedimiento para realizar contrataciones públicas en tiempos de declaratoria de emergencia, por lo cual, los entes administrativos de orden público, deben velar porque se cumpla a cabalidad, los procedimientos establecidos en la ley, con la finalidad de evitar delitos, relacionados al peculado y corrupción, por tanto, se recurre a la contraloría como órgano instituido, para generar averiguaciones y sanciones según sea el caso, aunado a la ciudadanía que debe organizarse para hacer el rol contralor sobre los bienes colectivos.

\section{DISCUSIÓN}

Se analizó el flagelo de la corrupción como consecuencia de la comisión de los delitos que atentan contra la eficiencia y buena marcha de la administración pública, los bienes y fondos públicos que forman parte del erario de la colectividad y que son custodiados por el Estado a través de sus diferentes órganos y entes que designan servidores y funcionarios públicos para proteger dicho patrimonio, socavan la confianza depositada en ellos, infringiendo la ley para apoderarse de los bienes que tienen bajo su tutela bien sea para sí mismos o en favor de terceras personas. 


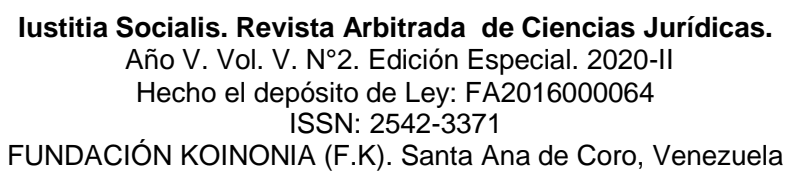

Alison Joyce Zapata Benavides; Julio César de Jesús Arrias-Añez; Juan Carlos Aradia Zambrano

En este orden de ideas, se han instalado en éste país diversas estructuras de corrupción con tentáculos en la administración pública a través de varios entramados como parte de la delincuencia organizada que pretende desfalcar el tesoro de todos los ecuatorianos, cada vez es más notable la acción delictiva orientada a sustraer, disponer, transferir o trasladar los fondos públicos destinados a satisfacer las necesidades básicas de la población en áreas tan sensibles como la Educación, Salud, Alimentación, Vialidad, Servicios Públicos, Seguridad Social, Infraestructura.

Siguiendo en el orden, la aparición del virus COVID 19 que generó una pandemia y como consecuencia una crisis de emergencia sanitaria, ameritó que se dictara el Estado de Excepción en el país que originó la implementación de procesos de contratación pública bajo la modalidad de adjudicaciones directas para la adquisición de equipos de bioseguridad, material y equipos médicos y hospitalarios, insumos, medicamentos, dejó al descubierto una serie de irregularidades que transgredían la normativa legal vigente en la materia, convirtiéndonos en referencia negativa a nivel mundial, siendo que este tipo de situación propicia corrupción en la administración pública latinoamericana (Madrid-Valerio \& Palomino-Ramírez, 2020).

En este escenario, resulta contraproducente para el Estado ecuatoriano la existencia de una corrupción ya institucionalizada productora de delitos contra la eficiencia de la administración pública con una incidencia alta del delito de peculado, al mismo tiempo resulta lesivo el costo en términos económicos y sociales que tiene para el país el problema del delito de peculado, pues este afecta directamente a la población ya que, este dinero que se fuga de las arcas del Estado forma parte de toda la colectividad, siendo considerable tener en cuenta que de acuerdo al derecho internacional, los delitos de peculado contra el bien común, no prescriben, para lo cual, debe existir voluntad política para ser sancionados (Vázquez-Vázquez, et al., 2020). 


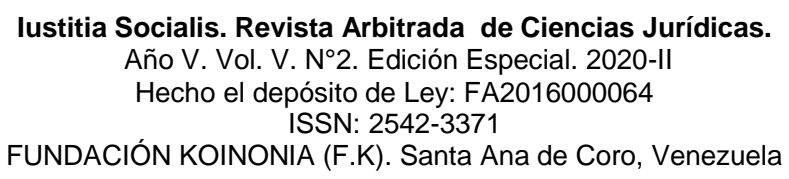

Alison Joyce Zapata Benavides; Julio César de Jesús Arrias-Añez; Juan Carlos Aradia Zambrano

\section{CONCLUSIONES}

Desde la fundamentación teórica se concluye que el delito de peculado es un tipo penal cuyo supuesto de hecho implica el abuso de confianza por parte del servidor público quien funge como garante de los fondos del Estado, quien dispone, traslada, sustrae los bienes que forman parte del patrimonio nacional en interés propio o de un tercero. Dicha acción dolosa, está tipificada en el artículo 278 del Código Orgánico integral Penal que data del año 2014, el mismo genera como efecto la corrupción que va vinculada muchas veces a la delincuencia organizada o a una asociación ilícita, tiene categoría de imprescriptible, por lo que, la acción para perseguirlo y las penas correspondientes no fenece, asimismo, puede ser juzgado en ausencia y es de orden público.

Desde el diagnóstico del estado actual, se concluye que la legislación ecuatoriana va en armonía con el contexto internacional en la materia de la eficiencia de la administración pública, resultando innovador al añadir la condición de: Imprescriptibilidad y ser juzgado en ausencia. Asimismo, las personas condenadas como consecuencia de éste tipo penal, no podrán ejercer cargos públicos y ser candidatos a cargos de elección popular. En este sentido, el delito de peculado tiene una alta incidencia de comisión en Ecuador, lo cual representa la falta de ética profesional y deontológica por parte de los infractores que ejercen posición de garantes de bienes del Estado, lo que implica una falta de consciencia y sensibilidad patriota.

El delito de peculado ocasiona una magnitud de daño gravosa, por su repercusión socioeconómica, al generar una pérdida de valores éticos - económicos por parte de los infractores y por las consecuencias que afectan a la colectividad que ven como su erario se deprecia y deprime y con esto el funcionamiento eficaz de estamentos imprescindibles para el desarrollo del buen vivir van en detrimento, lo cual redunda en la insatisfacción de necesidades básicas para la población, coyuntura que podría llevarnos a un Estado fallido y a la transgresión del Estado de Derecho. 


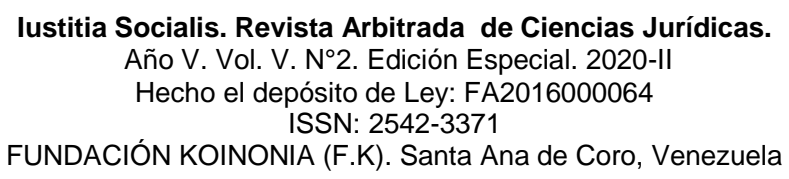

Alison Joyce Zapata Benavides; Julio César de Jesús Arrias-Añez; Juan Carlos Aradia Zambrano

\section{FINANCIAMIENTO}

No monetario.

\section{AGRADECIMIENTO}

A la Universidad Regional Autónoma de Los Andes, Quevedo; por incentivar el desarrollo de la Investigación.

\section{REFERENCIAS CONSULTADAS}

Asamblea Nacional de la República del Ecuador. (2014). Código Orgánico Integral Penal. [Comprehensive Organic Criminal Code]. Recuperado de https://n9.cl/g6sc

EI Universo (2020). IESS se depura por sospecha de sobreprecio en mascarillas. [IESS is purged due to suspicion of overpricing in masks]. Recuperado de https://n9.cl/9oizw

Encalada-Hidalgo, P. (2014). Teoría constitucional del delito y el código orgánico integral penal. [Constitutional theory of crime and the comprehensive organic penal code]. Tesis de maestría. Universidad Andina Simón Bolívar - Ecuador. Recuperado de http://repositorio.uasb.edu.ec/handle/10644/3745

Jalil-Ponce, J. (2019). Resolución N SECOB-SDG-2019-0075-R. Recuperado de https://n9.cl/d19lc

Jaramillo, S. J. (2019). Los delitos de cuello blanco "teoría de edwin sutherland.". [The white collar crimes "edwin sutherland theory"]. Sur Academia: Revista AcadémicaInvestigativa De La Facultad Jurídica, Social y Administrativa, 5(10), 44 - 50.

Kunicka-Michalska, B. (2017). La lucha contra la corrupción en América Latina. [The battle against corruption in Latin America]. Vectores de investigación, 12-13(1213), 197-211.

Ley Orgánica Del Sistema Nacional De Contratación Pública (2009). Sección II. Contrataciones en situaciones de emergencia. [Section II. Hiring in emergency situations]. Recuperado de https://n9.cl/day6n

Lopera-Echavarría, J, \& Ramírez-Gómez, C, \& Zuluaga-Aristazábal, M, \& Ortiz-Vanegas, J. (2010). El método analítico como método natural. Nómadas. Critical Journal of Social and Juridical Sciences, 25(1). 


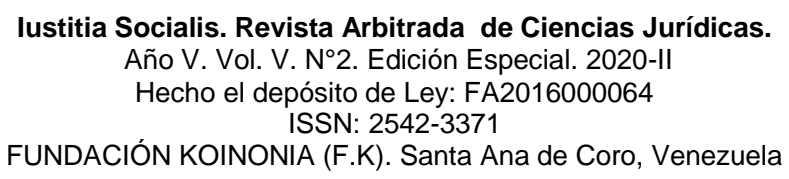

Alison Joyce Zapata Benavides; Julio César de Jesús Arrias-Añez; Juan Carlos Aradia Zambrano

Madrid-Valerio, C, \& Palomino-Ramírez, W. (2020). Oportunidades de corrupción y pandemia: el compliance gubernamental como un protector eficaz al interior de las organizaciones públicas. [The pandemic and opportunities for corruption: government compliance as an effective protection within public sector organizations]. Desde el Sur, 12(1), 213-239.

Quintero, T. (2018). Qué significa el delito de peculado y por qué afecta a los ciudadanos [What does the crime of embezzlement mean and why does it affect citizens?]. El Universo. Recuperado de https://n9.cl//9hw

Vázquez-Vázquez, F., Narváez-Zurita, C., Guerra-Coronel, M., \& Erazo-Álvarez, J. (2020). La imprescriptibilidad de los delitos contra la administración pública: Caso ecuatoriano. [The imprescriptibility of crimes against public administration: Ecuadorian case]. IUSTITIA SOCIALIS, 5(8), 208-224. http://dx.doi.org/10.35381/racji.v5i8.570 